\title{
Sun Protection Behaviors of Melanoma Survivors
}

\author{
Deborah Mayer, PhD, RN, AOCN, FAAN[Associate Professor], Annah Layman, PhD(c) \\ [Research Assistant], and John Carlson, MS[Research Associate Professor] \\ School of Nursing University of North Carolina at Chapel Hill
}

\begin{abstract}
Background-In the US, almost 800,000 individuals have had melanoma. They have a risk of reoccurrence and 5-10\% will develop new melanomas.

Objective-The purpose was to use a US population based sample of melanoma survivors and controls to evaluate sun protection behaviors.

Methods-We conducted a secondary data analysis of the 2005 and 2007 National Cancer Institute's Health Information National Trends Surveys. Skin protection behaviors of melanoma survivors $(\mathrm{N}=156)$ were compared to respondents without cancer $(\mathrm{N}=11408)$. We used unconditional logistic regression to estimate proportional odd ratios (OR) and 95\% confidence intervals (CI) adjusting for age, gender, race/ethnicity, and education.
\end{abstract}

\begin{abstract}
Results-Among melanoma survivors, $51.0 \%$ used sunscreen, $43.0 \%$ sought shade, and $74.3 \%$ wore protective clothing on sunny days. Among cancer-free controls, $34.7 \%$ used sunscreen, $37.1 \%$ sought shade, and $68.0 \%$ wore protective clothing. The adjusted ORs (95\% CIs) for sunscreen use and seeking shade in melanoma survivors compared to controls were 2.02 (1.36, $2.99)$ and $1.51(1.02,2.23)$, respectively. Further, 10 melanoma survivors reported using tanning beds after their diagnosis.
\end{abstract}

Limitations-HINTS questions did not ascertain skin self-exam practices, physician advice or knowledge about risk avoidance.

Conclusions-Melanoma survivors tend to use sunscreen and seek shade more frequently than cancer-free individuals but lower than expected given their cancer history. Health promotion programs among melanoma survivors need to continue to promote sunscreen and shade use, as well as discourage tanning bed use.

\section{Keywords}

melanoma; tanning; sun protection; tanning beds; survivorship; cancer prevention \& control; health behaviors

\section{Introduction}

The incidence of melanoma has increased over the last 20 years, especially among younger women. ${ }^{1}$ Between 1975 and 2007, overall melanoma incidence in the US increased 4.6\% and there are almost 800,000 melanoma survivors in the US. The majority $(84 \%)$ of those

\footnotetext{
Corresponding author mailing address: Dr. Deborah Mayer University of Chapel Hill CB7460 Chapel Hill, NC 5799-7460 dmayer@unc.edu Cell: 508-272-5482 Fax: 919-843-9900.

This paper is based on a poster presentation at the National Cancer Institute/CDC/LAF/American Cancer Society's $5^{\text {th }}$ Biennial Cancer Survivorship Research Conference, June 18, 2010, Washington DC.

Financial disclosures: None to declare
} 
diagnosed with melanoma are localized, conferring a relative 5-year survival rate of $98 \%$. This excellent prognosis, however, can be reduced by the development of new primary melanomas or other cancers, which can be $32 \%$ higher than those without melanoma. ${ }^{2,3}$

Exposure to ultraviolet (UV) radiation, whether directly from the sun or other sources, and the sensitivity of an individual's skin are risk factors for skin cancer and underscore 'sun safety' or 'sun smart' recommendations. ${ }^{4}$ These recommendations include covering up with wearing clothing, hats, and sunglasses that protect from UV radiation, using sun screen, seeking shade, and avoiding midday sun and other sources of UV radiation such as tanning beds. ${ }^{5}$ Previous studies of melanoma survivors have demonstrated mixed results regarding the adoption of sun protection behaviors. In a Canadian study, 217 melanoma survivors who were an average of six years after their diagnosis were surveyed about sun-awareness and protection. ${ }^{6}$ This descriptive study reported an increase in advice received, knowledge gained and behaviors changed after diagnosis. In another study of 229 melanoma survivors with a mean of 2.3 years after their diagnosis, most $(88.4 \%)$ conducted skin self exams (SSE) at least yearly but only only practiced moderate sun protection. ${ }^{7}$ However, in a small study comparing melanoma survivors with controls (35 in each group), Lee et al found that survivors had more knowledge of sun protection but time spent outdoors and percent experiencing sunburns were equivalent between the two groups. ${ }^{8}$ Less than half of the survivors reported being counseled about sun protection from their physician. In another study of melanoma survivors $(n=115)$ who were interviewed about skin self-exam and sun protection behaviors $9-13$ months after diagnosis, only $23 \%$ regularly used sun protection and $17 \%$ conducted SSE. 10 Given the discrepant results and relatively small and geographically limited samples of previous studies, the purpose of this study was to use a US population based sample of melanoma survivors and controls to evaluate sun protection behaviors.

\section{Methods}

\section{Data Source}

We conducted a secondary data analysis of the 2005 and 2007 National Cancer Institute's (NCI) Health Information National Trends Survey (HINTS) ${ }^{9}$ Initiated in 2003, HINTS is a biennial population-based survey to ascertain health information seeking practices and related lifestyle behaviors among adults over the age of 18 in the United States. HINTS is administered using random digit dialing, except in 2007when about half of completed surveys were conducted via mail. The total number of respondents participating in HINTS (and response rates) was 5586 respondents (20.8\%) in 2005 and 7674 respondents (24.2\% for random digit dialing and $31 \%$ for mailing) in $2007 .{ }^{10,11}$ Questions on lifestyle information includes questions on skin protection, exercise, diet, and tobacco use. Demographic and clinical information specific to cancer survivors is also collected using the HINTS tool. For more information on HINTS please see http://hints.cancer.gov/. Since this was a secondary data analysis and not considered human research, an exemption was obtained from the UNC Institutional Review Board.

The present analysis focuses on skin protection behaviors among melanoma survivors compared to cancer-free controls. Because information on skin protection behaviors was not collected in 2003, only data from the 2005 and 2007 HINTS was used. ${ }^{12}$ Between these two surveys, we identified 156 HINTS respondents who reported a history of melanoma. For controls, we considered all respondents who reported never having any type of cancer $(\mathrm{N}=11408)$.

Skin protection behaviors included sunscreen use, seeking shade, wearing protective clothing, and using tanning beds. Specific HINTS questions were as follows: 
When you go outside for more than 1 hour on a warm, sunny day...

- How often do you wear sunscreen?

- How often do you stay in the shade or under an umbrella?

- How often do you wear a shirt with sleeves that cover your shoulders?

- How often do you wear long pants?

- How often do you wear a hat?

How many times in the past 12 months have you used...

- Sunless tanning creams or sprays, also known as self-tanning or fake tanning?

- A tanning bed or booth?

Individuals who reported using sunscreen "always" or "often" or "did not go outside on sunny days" were considered to use sunscreen use as a protective behavior, while individuals who reported using sunscreen "sometimes," rarely," or "never" were classified as not using sunscreen. Likewise, individuals who reported seeking shade "always" or "often" or "did not go outside on sunny days" were considered to seek shade as a protective behavior, while individuals who reported seeking shade "sometimes," rarely," or "never" were classified as not seeking shade. Responses to questions on wearing a shirt with sleeves, long pants, and hats were collapsed into one variable. Individuals who reported "always" or "often" wearing long sleeves or long pants or hats or who reported "did not go outside on sunny days" were considered to wear protective clothing; all others were considered to not wear protective clothes.

\section{Statistical Analyses}

Using the multi-level and dichotomous variables described above, we calculated the frequency of sunscreen, shade and protective clothing use among melanoma survivors (cases) and cancer-free respondents (controls). Odd ratios (OR) and 95\% confidence intervals (CI) were then estimated using logistic regression. Models were adjusted for age, gender, race/ethnicity, and education level. Sample weights calculated by the NCI were used to produce population estimates. Replicate weights produced by the NCI were used in making jackknife estimates of standard errors.

\section{Results}

Demographic characteristics of melanoma survivors and cancer-free controls are summarized in table 1. Briefly, there were an approximately equal number of male and female melanoma survivors. Among controls, there were more females than males (62.8\% vs. 37.3\%). As expected, the majority of melanoma survivors were above the age of 50 $(86.5 \%)$, while controls had a higher proportion of younger ages. The majority of cases and controls were white (91\% and 72.8\%, respectively). Education level appeared similar between melanoma survivors and cancer-free controls with few individuals who did not graduated high school and similar proportions of individuals who graduated high school, attended some or graduated college. Likewise, body mass index (BMI) appeared similar between the two groups with few underweight individuals and approximately equal proportions of normal weight, overweight and obese individuals. The mean (standard deviation) time since cancer diagnosis was 2.9 (1.1) years with a range of $1-4$ years among melanoma survivor respondents,

The distribution of how often melanoma survivors and cancer-free controls practiced skin protection behaviors (sunscreen use, seeking shade and wearing protective clothing), as well 
as engaged in behaviors considered risk factors for melanoma (tanning bed use and tanning products use), are provided in table 2. For analytical purposes, levels of sunscreen use, seeking shade and wearing protective clothing were collapsed and will be discussed in detail later. For tanning beds and tanning products, a large portion of melanoma survivors reported not participating in such activities in the past 12 months ( $46.8 \%$ and $44.2 \%$, respectively). However, 10 melanoma survivors $(6.4 \%)$ did report using tanning beds and 21 melanoma survivors (13.5\%) did report using tanning products in the past 12 months which was after their diagnosis. The distribution of tanning bed and products use among melanoma survivors was similar to cancer-free controls, of whom $7 \%$ reported using tanning beds and $13.1 \%$ reported using tanning products in the last 12 months. The large portion of missing information on tanning bed and product use largely resulted from the 2007 survey and it is uncertain why this information was not fully ascertained during that specific year.

Table 3 presents the dichotomized variables for sunscreen use, seeking shade, and wearing protective clothing. Among melanoma survivors, $51 \%$ used sunscreen, $43 \%$ sought shade, and $74.3 \%$ wore protective clothing on sunny days. Among cancer-free controls, $34.7 \%$ used sunscreen, $37.1 \%$ sought shade, and $68.0 \%$ wore protective clothing. Table 3 also presents weighted crude and adjusted ORs (95\% CIs) for each skin protection behavior. Compared to crude estimates, adjustment for age, gender, race/ethnicity and education level only slightly altered estimates for sunscreen and shade use, but appeared to have a larger effect for protective clothing. The adjusted ORs (95\% CIs) for always/often sunscreen use and always/ often seeking shade in melanoma survivors compared to no cancer controls were 2.02 (1.36, $2.99)$ and $1.51(1.02,2.23)$, respectively, suggesting higher use of sunscreen and shade among melanoma survivors. However, these data do not support a significant difference between melanoma survivors and controls for always/often wearing protective clothing with an adjusted OR was $0.89(0.57,1.39)$.

\section{Discussion}

In this population based study, melanoma survivors appeared to have a higher odds of sunscreen usethan controls. Yet, less than 50\% of melanoma survivors reported frequent sunscreen use and sun avoidance, which is lower than other studies; Frieman and colleagues found $72 \%$ of melanoma survivors used sunscreen, ${ }^{6}$ while $59.4 \%$ did in Manne's study ${ }^{7}$ and $57 \%$ in Mujumdar's study ${ }^{9}$. Similar to sunscreen use, melanoma survivors had increased odds of seeking shade, but less than 50\% of melanoma survivors reported frequently seeking shade or avoiding the sun. Given the high risk for new skin cancers, one would expect higher adherence rates than those reported. Unlike other behaviors, the odds of wearing protective clothing did not vary by history of melanoma in HINTS and 70\% of melanoma survivors reported wearing long pants, long-sleeved shirts or hats. Of other clinical significance is the finding that 10 melanoma survivors reported using tanning beds after their cancer diagnosis which highlights Lee et al's ${ }^{8}$ report that $27 \%$ of the melanoma survivors have positive or neutral attitudes about tanning.

Previous studies have also demonstrated increased physician advice ${ }^{6}$ and increased knowledge ${ }^{7}$ about risk avoidance among melanoma survivors compared to controls, but information on these areas were not ascertained in the HINTS study. Information on other protective and early detection behaviors, such as the performance of skin self exam, were also not available. Further limitations of this study include that all demographic and behavioral information, including cancer status, was self-reported and not verified using external sources. However, major strengths of this study include the large, US-representative sample, systematic data collection, use of a control group, and available information on a variety of demographic and sun protection behaviors. 
As the incidence of melanoma continues to increase, the number of melanoma survivors will also grow. Many survivors will continue to be followed by their primary care provider, dermatologist or surgeon. These medical professionals need to continue health promotion programs among melanoma survivors to promote sun protection behaviors and discourage tanning bed use. Given the results of this study and others, increased awareness of the vulnerability and needs of this cohort of cancer survivors is warranted.

\section{Acknowledgments}

Funding: This work was supported by the National Cancer Institute (R03CA136077).

\section{References}

1. Altekruse, SF.; Kosary, CL.; Krapcho, M., et al., editors. SEER Cancer Statistics Review, 19752007. National Cancer Institute; Bethesda, MD: [Accessed July 21, 2010]. Available at http://seer.cancer.gov/csr/1975_2007/.

2. Spanogle J, Claarke C, Aroner S, Swetter S. Risk of second primary malignancies following cutaneous melanoma diagnosis: a population based study. J Am Acad Dermatol. 2010; 62(5):757767. [PubMed: 20223559]

3. Crocett E, Guzzinati S, Paci E, et al. The risk of developing a second, different, cancer among 14,650 survivors of the malignant cutaneous melanoma: a study by AIRTUM (the Italian Network of Cancer Registries). Melanoma Research. 2008; 18:230-234. [PubMed: 18477899]

4. NCI PDQ Skin Cancer Prevention. [Accessed July 21, 2010]. Available at http://www.cancer.gov/cancertopics/pdq/prevention/skin/HealthProfessional/page2\#Section_29

5. American Cancer Society. Skin cancer prevention. [Accessed July 21, 2010]. Available at http://www.cancer.org/docroot/PED/content/ ped_7_1_Skin_Cancer_Detection_What_You_Can_Do.asp?sitearea=PED.

6. Freiman A, Yu J, Loutfi A, Wang B. Impact of melanoma diagnosis on sun-awareness and protection: efficacy of education campains in a high-risk population. J Cutan Med Surg. 2004; 8(5): 303-309. [PubMed: 15868284]

7. Manne S, Lessin S. Prevalence and correlates of sun protection and skin self-examination practices among cutaneious malignant melanoma survivors. J Beh Med. 2006; 29(5):419-434.

8. Lee T, Brazier A, Shoveller J, Gallagher R. Sun-related behavior after a diagnosis of cutaneous malignant melanoma. Melanoma Research. 2007; 17:51-55. [PubMed: 17235242]

9. Cantor, D.; Coa, K.; Crystal-Mansour, S.; Davis, T.; Dipko, S.; Sigman, R. Health Information National Trends Survey (HINTS) 2007 Final Report. 2009 [Accessed July 21, 2010]. Available at http://hints.cancer.gov/docs/HINTS2007FinalReport.pdf

10. Cantor, D.; Covell, J.; Davis, T.; Park, I.; Rizzo, L. 2005 [Accessed July 21, 2010]. Health Information National Survey 2005 (HINTS 2005) Final Report. Available at http://hints.cancer.gov/docs/HINTS_2005_Final_Report.pdf

11. National Cancer Institute Division of Cancer Control and Population Sciences. HINTS Facts. [Accessed July 21, 2010]. Available at http://hints.cancer.gov/docs/HINTS_Fact_Sheet_accessible.pdf

12. National Cancer Institute Division of Cancer Control and Population Sciences. Cancer Communication Health Information National Trends Survey 2003 and 2005. [Accessed July 21, 2010]. Available at http://hints.cancer.gov/docs/hints_report.pdf 
Table 1

Demographics of Melanoma Survivors $(\mathrm{N}=156)$ and Cancer-Free Controls $(\mathrm{N}=11408)$ from the 2005 and 2007 HINTS

\begin{tabular}{|c|c|c|c|c|}
\hline \multirow[b]{2}{*}{ Demographic } & \multicolumn{2}{|c|}{ Melanoma Survivors ${ }^{*}$} & \multicolumn{2}{|c|}{ Cancer-Free Controls $^{* *}$} \\
\hline & $\mathbf{N}$ & $\%$ & $\mathbf{N}$ & $\%$ \\
\hline \multicolumn{5}{|l|}{ Sex } \\
\hline Male & 74 & 47.44 & 4248 & 37.24 \\
\hline Female & 82 & 52.56 & 7158 & 62.75 \\
\hline Missing & 0 & 0.00 & 2 & 0.02 \\
\hline \multicolumn{5}{|l|}{ Age } \\
\hline $18-34$ & 4 & 2.56 & 2049 & 17.96 \\
\hline $35-49$ & 16 & 10.26 & 3071 & 26.92 \\
\hline $50-64$ & 62 & 39.74 & 3438 & 30.14 \\
\hline $65-74$ & 41 & 26.28 & 1551 & 13.60 \\
\hline$<75$ & 32 & 20.51 & 1230 & 10.78 \\
\hline Missing & 1 & 0.64 & 69 & 0.60 \\
\hline \multicolumn{5}{|l|}{ Race and Ethnicity } \\
\hline White & 142 & 91.03 & 8301 & 72.76 \\
\hline African American & 6 & 3.85 & 1035 & 9.07 \\
\hline Hispanic & 4 & 2.56 & 1043 & 9.14 \\
\hline American Indian/Alaska Native & 5 & 0.64 & 120 & 1.05 \\
\hline Asian & 0 & 0.00 & 301 & 2.64 \\
\hline Native Hawiian/Pacific Islande & 0 & 0.00 & 24 & 0.21 \\
\hline Mutiple Races & 1 & 0.64 & 212 & 1.86 \\
\hline Missing & 2 & 1.28 & 372 & 3.26 \\
\hline \multicolumn{5}{|l|}{ Education } \\
\hline Less than high school & 11 & 7.05 & 1168 & 10.24 \\
\hline High school graduate & 38 & 24.36 & 2846 & 24.95 \\
\hline Some college & 53 & 33.97 & 3304 & 28.96 \\
\hline College graduate & 53 & 33.97 & 3869 & 33.91 \\
\hline Missing & 1 & 0.64 & 221 & 1.94 \\
\hline \multicolumn{5}{|l|}{ BMI } \\
\hline Underweight & 4 & 2.56 & 220 & 1.93 \\
\hline Normal weight & 50 & 32.05 & 3956 & 34.68 \\
\hline Overweight & 51 & 32.69 & 3805 & 33.35 \\
\hline Obese & 45 & 28.85 & 2942 & 25.79 \\
\hline Missing & 6 & 3.85 & 485 & 4.25 \\
\hline
\end{tabular}


Table 2

Frequency of Skin Protection Behaviors among Melanoma Survivors (N=156) and Cancer-Free Controls $(\mathrm{N}=11480)$ from the 2005 and 2007 HINTS

\begin{tabular}{|c|c|c|c|c|}
\hline \multirow[b]{2}{*}{ Behavior } & \multicolumn{2}{|c|}{ Melanoma Survivors* } & \multicolumn{2}{|c|}{ Cancer-Free Controls $^{* *}$} \\
\hline & $\mathbf{N}$ & $\%$ & $\mathbf{N}$ & $\%$ \\
\hline \multicolumn{5}{|l|}{ Sunscreen ${ }^{\dagger}$} \\
\hline Always & 39 & 25.00 & 1667 & 14.52 \\
\hline Often & 33 & 21.15 & 2051 & 17.87 \\
\hline Sometimes & 30 & 19.23 & 2673 & 23.28 \\
\hline Rarely & 23 & 14.74 & 2010 & 17.51 \\
\hline Never & 23 & 14.74 & 2700 & 23.52 \\
\hline Do not go outside on sunny days & 7 & 4.49 & 212 & 1.85 \\
\hline Missing & 1 & 0.64 & 95 & 0.83 \\
\hline \multicolumn{5}{|l|}{ Shade $\neq$} \\
\hline Always & 20 & 12.82 & 1179 & 10.27 \\
\hline Often & 44 & 28.21 & 2894 & 25.21 \\
\hline Sometimes & 46 & 29.49 & 4067 & 35.43 \\
\hline Rarely & 21 & 13.46 & 1842 & 16.05 \\
\hline Never & 18 & 11.54 & 1092 & 9.51 \\
\hline Do not go outside on sunny days & 0 & 0.00 & 52 & 0.45 \\
\hline Missing & 7 & 4.49 & 282 & 2.46 \\
\hline \multicolumn{5}{|l|}{ Clothing $\S$} \\
\hline Always & 71 & 45.51 & 4615 & 40.20 \\
\hline Often & 39 & 25.00 & 2943 & 25.64 \\
\hline Sometimes & 26 & 16.67 & 2296 & 20.00 \\
\hline Rarely & 9 & 5.77 & 709 & 6.18 \\
\hline Never & 3 & 1.92 & 576 & 5.02 \\
\hline Do not go outside on sunny days & 0 & 0.00 & 47 & 0.41 \\
\hline Missing & 8 & 5.13 & 222 & 1.93 \\
\hline \multicolumn{5}{|l|}{ Tanning Beds\# } \\
\hline Otimes & 73 & 46.79 & 4400 & 38.33 \\
\hline $1-2$ times & 1 & 0.64 & 180 & 1.57 \\
\hline $3-10$ times & 4 & 2.56 & 320 & 2.79 \\
\hline $11-24$ times & 3 & 1.92 & 154 & 1.34 \\
\hline 25 times & 2 & 1.28 & 147 & 1.28 \\
\hline Missing & 73 & 46.79 & 6207 & 54.07 \\
\hline \multicolumn{5}{|l|}{ Tanning Products $\dagger \dagger$} \\
\hline Otimes & 69 & 44.23 & 4189 & 36.49 \\
\hline $1-2$ times & 2 & 1.28 & 528 & 4.60 \\
\hline $3-10$ times & 6 & 3.85 & 530 & 4.62 \\
\hline 11-24 times & 8 & 5.13 & 242 & 2.11 \\
\hline 25 times & 5 & 3.21 & 203 & 1.77 \\
\hline
\end{tabular}




\begin{tabular}{|c|c|c|c|c|}
\hline \multirow[b]{2}{*}{ Behavior } & \multicolumn{2}{|c|}{ Melanoma Survivors* } & \multicolumn{2}{|c|}{ Cancer-Free Controls $^{* *}$} \\
\hline & $\mathbf{N}$ & $\%$ & $\mathbf{N}$ & $\%$ \\
\hline Missing & 66 & 42.31 & 5716 & 49.79 \\
\hline \multicolumn{5}{|c|}{ Self-reported a history of melanoma } \\
\hline \multicolumn{5}{|c|}{ *** Self-reported never having a history of any cancer } \\
\hline \multicolumn{5}{|c|}{${ }^{\dagger}$ Self-reported sunscreen use when going outside for more than 1 hour on a warm, sunny day } \\
\hline Self-reporte & utside fo & than 1 hou & on a warn & y day \\
\hline
\end{tabular}


Table 3

Odds of Skin Protection Behaviors among Melanoma Survivors (N=156) Compared to Cancer-Free Controls $(\mathrm{N}=11480)$ from the 2005 and 2007 HINTS

\begin{tabular}{|c|c|c|c|c|}
\hline Behavior & Melanoma Survivors N & Cancer-Free Controls N & $\begin{array}{c}\text { Unadjusted OR }(95 \% \\
\text { CI })^{*}\end{array}$ & $\begin{array}{c}\text { Adjusted OR }(95 \% \\
\text { CI })\end{array}$ \\
\hline \multicolumn{5}{|l|}{ Sunscreen ${ }^{\dagger}$} \\
\hline Always/Often & 79 & 3930 & $2.06(1.41,3.01)$ & $2.02(1.36,2.99)$ \\
\hline Sometimes/Rarely/Never & 76 & 7383 & 1.0 & 1.0 \\
\hline Missing & 1 & 95 & & \\
\hline \multicolumn{5}{|l|}{ Shade $^{\dagger}$} \\
\hline Always/Often & 64 & 4125 & $1.58(1.08,2.33)$ & $1.51(1.02,2.23)$ \\
\hline Sometimes/Rarely/Never & 85 & 7001 & 1.0 & 1.0 \\
\hline Missing & 7 & 282 & & \\
\hline \multicolumn{5}{|l|}{ Clothing $\S$} \\
\hline Always/Often & 110 & 7605 & $1.35(0.88,2.08)$ & $0.89(0.57,1.39)$ \\
\hline Sometimes/Rarely/Never & 38 & 3581 & 1.0 & 1.0 \\
\hline Missing & 8 & 2222 & & \\
\hline
\end{tabular}

Abbreviations: OR odds ratio CI confidence interval

* Odds ratios weighted based on population estimates from the National Cancer Institute

** Odds ratios weighted based on population estimates from the National Cancer Institute and adjusted for age, gender, race/ethnicity, and education level

${ }^{\dagger}$ Self-reported sunscreen use when going outside for more than 1 hour on a warm, sunny day

${ }^{*}$ Self-reported shade use when going outside for more than 1 hour on a warm, sunny day

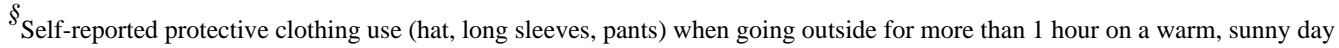

